\title{
EXPLORANDO PERCEPCIONES DEL IMPACTO DEL CAMBIO CLIMÁTICO EN TRES REGIONES EN EL PERÚ
}

\author{
Paola Alejandra Torres-Slimming \\ Universidad Peruana de Ciencias Aplicadas \\ ORCID: 0000-0001-9741-9470 \\ Lucia López Florez \\ INTE-PUCP \\ ORCID: 0000-0002-1564-7363
}

Karina Castañeda Checa

INTE-PUCP

ORCID: 0000-0001-7993-4081

Oscar Durand Galarza

Periodista Visual Independiente

Paula Skye Tallman

Universidad Loyola de Chicago, Estados Unidos de América

ORCID: 0000-0003-2384-249X

\section{Gabriela Salmón Mulanovich}

INTE-PUCP

ORCID: 0000-0002-8918-7689

\begin{abstract}
Resumen: El cambio climático es atribuido directa o indirectamente a la actividad humana y se considera como la mayor amenaza a la salud pública del siglo XXI. Tiene impactos sobre el acceso al agua, la alimentación, en la agricultura y medios de vida, y en la salud de las personas. El objetivo de este texto es evaluar las percepciones del cambio climático en niños, niñas, adolescentes, autoridades y representantes de organizaciones juveniles, de mujeres y que trabajan con la niñez en las regiones de Cusco, Loreto y Piura, en Perú. Para ello se realizó un estudio cualitativo que utilizó la técnica de Fotovoz con doce adolescentes y entrevistas a profundidad con veintidós actores clave. Para el análisis se utilizaron las narrativas en las fotos y el análisis temático de las entrevistas. En las tres regiones se identificaron cambios extremos en el clima. En la región
\end{abstract}


de Cusco reconocieron el cambio de temperatura con sequías y heladas, mientras que en Loreto y Piura manifestaron aumento en la intensidad de lluvias. En general, estos cambios afectaron la agricultura. El cuidado del agua como recurso, tanto en su acceso y calidad, fue una gran preocupación para todos los participantes. El estudio concluye que no se identifica el concepto de cambio climático como tal, sin embargo, describen cambios y variaciones en el clima que afectan sus actividades diarias, en particular la agricultura. Un tema transversal a todas las regiones fue el cuidado y uso del recurso del agua. Además, los jóvenes se mostraron particularmente entusiasmados en colaborar en iniciativas futuras contra el cambio climático.

Palabras clave: Cambio climático, Percepciones locales, Fotovoz, Perú, Poblaciones vulnerables.

\title{
Exploring Perceptions of the Impact of Climate Change in three Regions in Peru
}

\begin{abstract}
Climate change is directly or indirectly attributed to human activity and is considered the greatest threat to public health of the 21st century. It has impacts on access to water, food, agriculture and livelihoods, and people's health. The objective of this article is to evaluate the perceptions of climate change in children, adolescents, authorities and representatives of youth organizations, women's organizations and organizations that work with children in the regions of Cusco, Loreto and Piura, in Peru. We conducted a qualitative study using Photovoice with twelve adolescents and in-depth interviews with twenty-two key stakeholders. For the analysis, thematic analysis of the narratives in the photos and of the interviews were used. Extreme changes in weather were identified in all three regions. In the Cusco region they recognized the change in temperature with droughts and frosts, while in Loreto and Piura they showed an increase in rain intensity. In general, these changes affected agriculture. The care of water as a resource, both in its access and quality, was a great concern for all the participants. The study concludes that the concept of climate change as such is not identified, however, they describe changes and variations in the
\end{abstract}


weather that affect their daily activities, particularly agriculture. A cross-cutting theme for all regions was the care and use of the water resource. In addition, young people were particularly enthusiastic about collaborating on future initiatives against climate change.

Keywords: Climate Change, Local Perceptions, Photovoice, Peru, Vulnerable Populations

\section{Paola Alejandra Torres-Slimming}

PhDc en Ciencias de la Vida por la Universidad Peruana Cayetano Heredia en colaboración con el Proyecto IHACC de la Universidad de Alberta, Canadá. Médica epidemióloga con interés y experiencia en el trabajo con comunidades vulnerables e indígenas. Su investigación se centra en la atención primaria, enfermedades infecciosas y la seguridad del agua, bajo el enfoque de Ecosalud

Correo: pcmeptor@upc.edu.pe

\section{Lucia López Flórez}

Magíster en Gobernanza y Desarrollo por la Universidad de Amberes, Bélgica, y licenciada en Comunicación para el Desarrollo por la Pontificia Universidad Católica del Perú. Ha sido docente de la Facultad de Ciencias y Artes de la Comunicación de la PUCP y es Coordinadora del Área de Investigación y Relaciones Institucionales del INTE-PUCP.

Correo: lucia.lopez@pucp.pe

\section{Karina Castañeda Checa}

Magíster en Gobierno y Ciencia Política y egresada de la Maestría en Comunicaciones ambos de la Pontificia Universidad Católica. Socióloga con interés y experiencia en investigación interdisciplinaria sobre desarrollo humano, empoderamiento, género y medio ambiente, especialmente en la Amazonía y en la zona andina peruana. Coordinadora del Área de Investigación y Relaciones Institucionales del INTE-PUCP.

Correo: kcastaneda@pucp.edu.pe 


\section{Oscar Durand Galarza}

Periodista Visual Independiente. Egresado del programa de fotoperiodismo del Instituto Tecnológico de Rochester, Estados Unidos. Periodista con experiencia en la producción de ensayos fotográficos y videos documentales. Su trabajo es publicado por periódicos, revistas y organizaciones humanitarias y se enfoca fundamentalmente en temas relacionados con la inmigración y el desplazamiento humano.

Correo: oscar@oscardurand.com

\section{Paula Skye Tallman}

BA en Biología del Comportamiento por la Universidad Johns Hopkins, PhD Antropología por la Universidad Northwestern y postdoctorado en The Field Museum of Natural History en Chicago. Es Profesora Asistente de Antropología en la Universidad Loyola de Chicago. Sus investigaciones examinan los impulsores de las inequidades en salud en América del Sur y Asia, con un enfoque en conectar con iniciativas de justicia social.

Correo: paulaskyetallman@gmail.com

\section{Gabriela Salmón Mulanovich}

BSc en Ciencias, Biología en la Universidad Nacional Agraria - La Molina. Tiene un doctorado en Salud Internacional en el área de Epidemiología y Control de Enfermedades Globales por la Escuela Bloomberg de Salud Pública de la Universidad de Johns Hopkins. Su investigación está enfocada en la epidemiología de las enfermedades infecciosas, su relación con el cambio climático y los cambios en el paisaje. Trabaja también aspectos de resistencia antimicrobiana con el enfoque de Una Salud (One Health) y la mejora de servicios de salud comunitarios. Es profesora por asignaturas y miembro de la Asamblea del INTE-PUCP.

Correo: gsalmonm@pucp.edu.pe 


\title{
1. Introducción
}

\begin{abstract}
En este lío nosotros no somos los provocadores; sin embargo, seremos las víctimas.
\end{abstract}

Representante de organización ambiental, Loreto

El cambio climático se atribuye a cambios en el clima a consecuencia de la emisión excesiva de gases de efecto invernadero por la actividad humana (MINAM 2010). Actualmente, estos cambios se han asociado a eventos climáticos extremos en diferentes partes del mundo (Watts et al. 2021). El Perú es uno de los países más vulnerables en Latinoamérica al cambio climático, particularmente al recurso del agua (Hardoy \& Romero Lankao 2011; MINAM 2016). En 2020 se reportaron a nivel global una serie de indicadores alarmantes en el seguimiento al cambio climático para las distintas zonas geográficas. Dentro de los indicadores se encuentran: i) disminución de la productividad general, atribuida a altas temperaturas, que impacta en la salud de las personas, ii) aumento de eventos climáticos extremos generando desastres, iii) mayor riesgo de exposición a incendios forestales, iv) bajo rendimiento y pérdidas de los cultivos con el consecuente impacto en la seguridad alimentaria (Watts et al. 2021). En 2021, el Perú ha renovado su Estrategia nacional ante el cambio climático al 2050, la cual intenta reforzar sus objetivos e incentivar la participación ciudadana (MINAM s.f.; MINAM 2015).

El Perú presenta diversas áreas geográficas con distintos pisos ecológicos que lo hacen particularmente vulnerable al cambio climático. Tiene zonas expuestas a sequías, desertificación, inundación, lugares propensos a desastres, ecosistemas de montaña frágiles, entornos urbanos con alta contaminación atmosférica, entre otros (MINAM 2015). Sumado a ello, la inequidad y pobreza acentúan las influencias directas generadas por el cambio climático, desencadenando dinámicas complejas como la migración y conflictos sociales (Andersen et al. 2009; Singer 2019). Dada la aceleración e intensificación de factores estresantes relacionados con el clima, particularmente en poblaciones vulnerables, es esencial comprender sus experiencias «sobre el terreno» para identificar un plan de adaptación y 
mitigación del cambio climático apropiado a nivel local (Hissa 2016; Wang \& Burris 1997).

En este contexto se desarrolló un estudio cualitativo en colaboración con Plan Internacional (PI) y el Instituto de la Naturaleza, Tierra y Energía de la Pontificia Universidad Católica del Perú (INTE-PUCP), (el cual presentamos) con el objetivo de recoger la percepción sobre los impactos identificados del cambio climático en los niños, niñas, adolescentes, jóvenes y mujeres. Esto se realizó a través del registro de las experiencias de los y las adolescentes, autoridades y representantes de organizaciones juveniles, de mujeres y que trabajan con la niñez en tres regiones muy diferentes en el país: Cusco, Loreto y Piura.

\section{Metodología}

La metodología empleada utilizó dos técnicas cualitativas de recopilación de datos: Fotovoz y entrevistas a profundidad con actores clave. El trabajo de recojo de información se realizó entre marzo y junio de 2021. Fotovoz es un método de investigación participativa que utiliza la fotografía como una herramienta (Hissa 2016) mediante la cual las personas pueden identificar, representar y dar a conocer alguna problemática desde su perspectiva (Wang \& Burris 1997). Los criterios de selección de los participantes se elaboraron de forma conjunta con PI. Se reclutó a 12 participantes adolescentes entre los 14 y17 años de edad, distribuidos equitativamente por género y por región, de zonas urbanas y periurbanas. Debían de haber colaborado con PI anteriormente, a manera de asegurar la conectividad durante las seis sesiones del taller de Fotovoz. Las regiones seleccionadas fueron Piura, Cusco, y Loreto, con la intención de que cada región tenga características ecológicas distintas. A cada uno de los doce adolescentes se le entregó un dispositivo electrónico portátil (Tablet) con el objetivo de identificar su percepción sobre los impactos del cambio climático en su quehacer diario. Además, como parte del taller de Fotovoz, recibieron entrenamiento en el uso de estos dispositivos y en aspectos introductorios sobre fotografía.

Para las entrevistas a profundidad se utilizó una guía de entrevistas semiestructuradas con la intención de profundizar en los impactos diferenciados, y lograr obtener la mayor información posible y de manera contextualizada. Se entrevistó a adultos (hombres y mujeres), autoridades del sector educativo, representantes de organizaciones locales, en particular de organizaciones sociales de adultos que trabajan temas de salud, derechos humanos, con énfasis en la niñez, adolescencia y mujeres; representantes del gobierno regional y local dedicados a la gestión de riesgos por cambio climático, gestión de riesgos por desastres, gestión del agua y gestión de 
la salud (ver tabla 1). También se entrevistó a cuatro representantes de organizaciones juveniles locales. En total, se realizaron 22 entrevistas diferenciadas equitativamente por género: 7 entrevistas en Cusco, 7 en Piura y 8 en Loreto. A continuación, en la tabla 1 se presenta la descripción general sobre los y las informantes según cada región.

Tabla 1. Participantes de entrevistas a profundidad

\begin{tabular}{c|c|c}
\hline No & Región & Representante \\
\hline 1 & Cusco & $\begin{array}{c}\text { Autoridad regional de recursos } \\
\text { hídricos }\end{array}$ \\
\hline 2 & Cusco & Autoridad regional de salud \\
\hline 3 & Cusco & Organización juvenil local \\
\hline 4 & Cusco & Organización juvenil local \\
\hline 5 & Cusco & Autoridad regional para agricultura \\
\hline 6 & Cusco & $\begin{array}{c}\text { Autoridad regional para desarrollo } \\
\text { social }\end{array}$ \\
\hline 7 & Cusco & Autoridad local para el ambiente \\
\hline 9 & Loreto & Organización ambiental local \\
\hline 10 & Loreto & Autoridad regional ambiental \\
\hline 11 & Loreto & Organización local de mujeres \\
\hline
\end{tabular}

\begin{tabular}{c|c|c}
\hline No & Región & Representante \\
\hline 12 & Loreto & Organización local dedicada a la niñez \\
\hline 13 & Loreto & Organización ambiental local \\
\hline 14 & Loreto & Autoridad regional de salud \\
\hline 15 & Loreto & Organización local para investigación \\
\hline 16 & Piura & Autoridad regional ambiental \\
\hline 17 & Piura & Organización juvenil local \\
\hline 18 & Piura & Autoridad local de desarrollo social \\
\hline 19 & Piura & Organización ambiental local \\
\hline 20 & Piura & Organización local de mujeres \\
\hline 21 & Piura & Organización ambiental local \\
\hline 22 & Piura & Autoridad local de educación \\
\hline
\end{tabular}

Los talleres de Fotovoz y las entrevistas fueron grabadas y posteriormente transcritas. En cuanto al análisis de la información, para las sesiones de Fotovoz se seleccionaron, por cada región, las fotografías e historias que mejor ejemplificaran las percepciones del cambio climático. Las entrevistas transcritas ingresaron a una matriz de doble entrada, agrupadas en códigos, categorías y ejes temáticos para el análisis temático. Los ejes temáticos seleccionados fueron: impactos generales del cambio climático; impacto en el acceso al agua y en el clima extremo; impacto en la alimentación, agricultura y medios de vida; impactos en la salud, fortalezas y acciones para enfrentar el cambio climático.

Debido al contexto de la pandemia por el SARS-CoV-2, las sesiones de Fotovoz y las entrevistas se realizaron de manera virtual mediante la plataforma Zoom, previo consentimiento del participante. En el caso de los menores de edad, se contó con el consentimiento del apoderado y el asentimiento del menor. Este estudio fue revisado y aprobado por el comité de ética de la PUCP: dictamen N 005-2021-CEI-CCSSHHyAA/PUCP, y de la oficina de Protección y Salvaguarda de PI. 


\section{Resultados}

El cambio climático es un concepto no reconocido aún por la mayoría de los entrevistados. Al explorar detenidamente las dimensiones que componen este concepto, se constató que identifican la expresión, pero no tienen incorporado su significado, mucho menos actividades de prevención. Sin embargo, reconocen ciertos cambios en los componentes del clima y el impacto que genera en sus vidas familiares y en comunidad.

En las tres regiones, los participantes coincidieron al reconocer impactos del cambio en el clima, cada región con sus propias particularidades. En general, identificaron la repercusión que tienen los eventos extremos del clima sequía, inundaciones, heladas y olas de calor - sobre los medios de vida de las comunidades, como la agricultura. Asimismo, identificaron graves problemas de contaminación con residuos sólidos debido a la quema de estos y por contaminación en recursos hídricos.

En la región Piura, todas las personas entrevistadas reconocieron cambios extremos de temperatura, el nivel e intensidad de la lluvia, y la variación de la producción agrícola. Estos cambios son visibles y perciben que están afectando tanto a las zonas urbanas como rurales. Un representante del Gobierno Regional también identificó a la deforestación como un factor que influye en el impacto local del cambio climático: «[...] en realidad, de por sí tenemos en Piura un problema de deforestación, de medio millón de hectáreas deforestadas, entonces eso también creo que influye en los efectos del cambio climático». Los adolescentes de Piura participantes de Fotovoz narraron historias de cómo el calor extremo y la sequedad en el ambiente han impactado en la productividad.

Figura 1

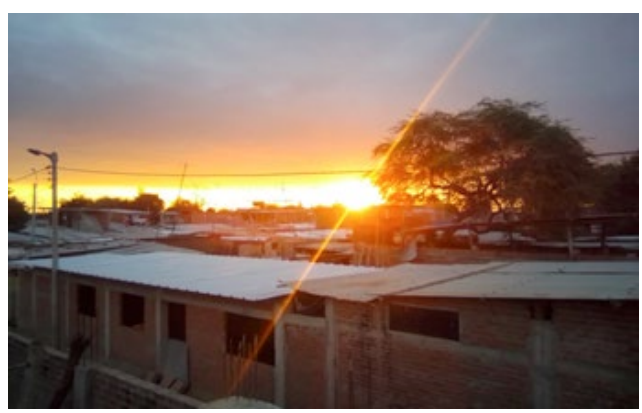

Fotografía tomada para el Taller de Fotovoz, sesión 3 del 8 de mayo, 2021.

Fotografía que narra el calor extremo en Piura: «Me refería al tema del cambio climático, de las estaciones de la comunidad, ¿no? Por ejemplo, aquí estamos observando una foto en la cual este es un atardecer. Y eso significa que en la tarde ha hecho mucho sol y ahí está, que ya se oculte el sol, ¿no? Ya se va a anochecer...» (adolescente, varón, 16 años, Piura). 
La mayoría de los informantes clave en Loreto reportaron percibir el incremento de la temperatura como característica principal del cambio climático. Otros atributos mencionados fueron el aumento de las precipitaciones y la contaminación del agua. Las y los adolescentes de Loreto reconocieron el impacto directo del aumento de la temperatura en su salud. Asimismo, mencionaron las crecidas de los ríos y las lluvias intensas que afectan los cultivos de las comunidades ribereñas o rurales, y perciben épocas con precipitaciones más intensas y menos predecibles.

Figura 2

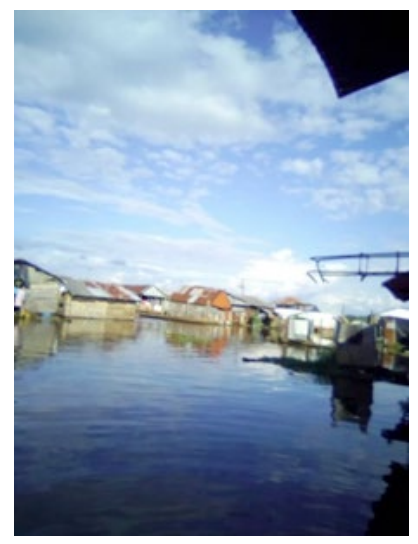

Fotografía tomada para el taller virtual de Fotovoz, sesión 3 del 8 de mayo, 2021.

Fotografía que narra el calor extremo en Loreto: «Y los techos de las calaminas ya como que arde y adentro... se calienta» (adolescente, varón, 17 años, Loreto).

En Cusco se percibe el cambio intenso en la temperatura y en las estaciones como una variación en el clima. Estos cambios son cada vez más frecuentes. Las y los adolescentes de esta región, reconocieron los efectos de las bajas temperaturas en sus vidas. Dan como ejemplo las sequías, que afectan el rendimiento de los cultivos. Sin el agua necesaria, las cosechas se secan y se pudren, generando pérdidas y reduciendo la productividad. En el caso de la región de Cusco, si bien cuentan con ciertos sistemas de almacenamiento de aguas en lagunas y canales, perciben escasez del recurso y sequías. 
Figura 3

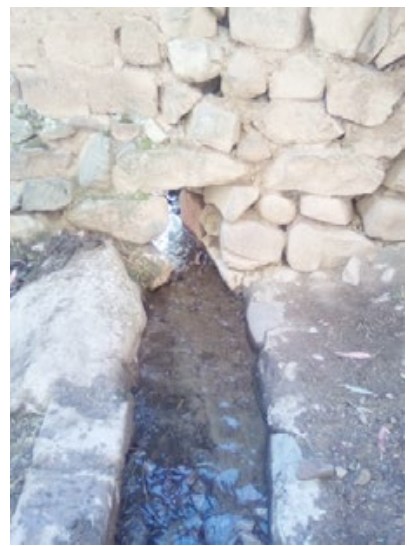

Fotografía tomada para el taller de Fotovoz, sesión 4, 15 de mayo, 2021.

Fotografía que muestra la situación de los canales en Cusco: «Y hay un sitio como reservorio, eso, que llama como agua potable y nada de ahí hay el agua, pero se desperdicia el agua» (adolescente, mujer, 16 años, Cusco).

Un tema común y transversal a todas las regiones es el cuidado del recurso del agua para el uso humano y agrícola.

Figura 4

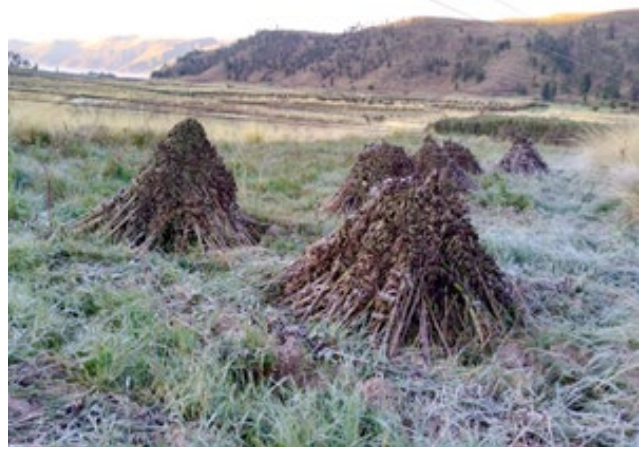

Fotografía tomada para el Taller de Fotovoz, sesión 3 del 8 de mayo, 2021.

Fotografía que muestra el impacto del clima en la agricultura: «En esta parte les quería mostrar al finalizar la cosecha de las habas... primeramente, lo juntamos para que la base seque y luego lo cosechemos solitas... lo hacemos antes de que empiece la helada, porque al momento que está creciendo, el avance en su desarrollo comienza a congelarse las puntas y el sol del día lo quema esa parte. Entonces... estamos perdiendo una parte de todo el trabajo, ¿no?» (adolescente, varón, 16 años, Cusco). 
En Cusco y Piura muchos entrevistados afirmaron que el cambio en la frecuencia e intensidad de las lluvias tiene un impacto directo en la agricultura. Al referirse a sus parcelas agrícolas familiares, un joven manifestó en los talleres de Fotovoz: «Se les seca. [...] Bueno, de no sembrar producto. De no poder ser, no poder comercializar». En estas dos regiones existe una gran preocupación relacionada con el estrés hídrico y la dificultad de acceder a las fuentes de agua.

En cuanto a Loreto, los participantes identificaron las fuertes lluvias e inundaciones de los ríos como amenazas para las comunidades ribereñas. En las entrevistas a profundidad, un representante de una organización ambiental local mencionó: «[...] cuando le preguntas a esas personas por qué ya no cultivan, es porque dicen que el río ya es totalmente impredecible». Además de amenazar los campos agrícolas, las fuertes lluvias y las sequías impactan directamente en la inseguridad del agua en las comunidades. Una respuesta a esta situación es la migración hacia las zonas urbanas o hacia zonas más altas. Sin embargo, esto no resuelve todos los problemas e incluso puede generar otras dificultades: «Entonces, eso también ha hecho que estas poblaciones estén desanimadas y cada vez menos gente esté apostando por los territorios aluviales, y migran a las zonas altas y esto trae cada vez mayor deforestación», explicó un antiguo funcionario del Gobierno Regional. Asimismo, el traslado hacia zonas urbanas, donde falta el agua potable y servicios de saneamiento, facilita la presencia de diarrea y enfermedades gastrointestinales en la población, según reportó una investigadora de un instituto de investigación local: «no hay una infraestructura de agua, ni desagüe, menos de alcantarillado». Además, los problemas con el agua están asociados con mayor riesgo de anemia y desnutrición en niños y niñas debido al estrecho vínculo con la inseguridad alimentaria y la sensibilidad de los niños pequeños a estos factores estresantes, según identificó un representante de la autoridad regional de salud: «[...] ahí la desnutrición o la anemia son las que podría incrementarse [...] Y todo eso está ligado a un comportamiento de los ríos amazónicos».

Estos problemas de acceso a poca o grandes cantidades de agua segura, impactan de manera diferenciada en cada región, en cada grupo etario, y están asociados a sus patrones culturales, patrones de identidades, sistemas de género, economías y salud. Dicha preocupación la manifestó claramente un representante de una organización juvenil de Piura en la entrevista a profundidad: «¿qué va a pasar con estos sistemas ancestrales de siembra y cosecha cuando no haya mano de obra? Justamente la crianza de agua viene de personas criando el territorio, pero si no hay personas, probablemente nuestra disponibilidad hídrica se llegue a reducir». 
Los impactos expresados por los participantes fueron diferenciados por regiones, particularmente en aquellos entrevistados que viven en comunidades dependientes de la naturaleza. Por ejemplo, en el caso de Loreto, las lluvias intensas con inundaciones obligan a grupos de familias a emigrar a zonas periurbanas, siendo los escolares los más afectados, con un alto índice de deserción escolar. Los niños, niñas y adolescentes se ven obligados a dejar la escuela por periodos indefinidos para trabajar. Las mujeres, por su parte, también se vuelven más vulnerables porque en su necesidad de trabajar, pueden exponerse a explotación laboral y, algunas veces, hasta sexual, de acuerdo a una representante de una organización local dedicada a la niñez: «[...] la salida de sus zonas rurales a la ciudad también va a ir llenando aquellos cinturones de pobreza [...] acceder muchas veces a trabajos o actividades injustas [...] de la explotación sexual, de la explotación laboral».

Finalmente, todos los adolescentes que participaron del taller de Fotovoz, así como los representantes de las organizaciones juveniles, mostraron una gran disposición para involucrarse con su entorno y su comunidad. Un adolescente, participante del taller de Fotovoz de Loreto señaló:

Los trabajos que nos envían [en la escuela] son acerca de qué contaminación nosotros tenemos aquí en Loreto [...] no sé si eso pasa porque no tienen información [...] y la capacidad, ¿no? Y eso me hace pensar muchas cosas, ¿no? Ya que estoy sacando mucho provecho de estar en unas organizaciones como esta [...] Me interesa mucho vivir, sea mi sociedad, y quiero que esto cambie, eso quería mostrarles, la problemática que es el cambio climático y cómo funciona el agua.

\section{Discusión}

El presente estudio tuvo como objetivo analizar las percepciones del cambio climático desde la perspectiva de los distintos participantes, adolescentes y adultos de tres regiones del Perú. Es importante destacar que los participantes no mostraron tener información extendida sobre el cambio climático. Solo algunos actores con conocimiento un poco más especializado podían hacer referencia a este problema. Estudios previos han señalado una situación similar sobre las percepciones del cambio climático en la cuenca amazónica (Funatsu et al. 2019; MINAM \& MIMP s.f.). Reportes previos también han reconocido la ausencia en la incorporación de este tema en políticas y actividades en los diversos sectores y de una perspectiva sistémica que permita abordar los retos que presenta el cambio climático en el territorio (Fiksel 2006). Esto puede ser un motivo por el cual no se destaca como un problema 
inmediato y tampoco se identifica el vínculo con otros desafíos urgentes como la deforestación (Sánchez \& Reyes 2015; Siclari 2021; Gatti et al. 2021). Sin embargo, al consultar por la manifestación de algunos atributos de la crisis climática de manera local, se reconocieron los impactos y las repercusiones en el entorno cercano y también efectos indirectos. Esto es similar a lo que se reporta en otras regiones (Cunsolo Willox et al. 2012; Ostapchuk et al. 2015).

Los contrastes en las tres diferentes regiones fueron muy evidentes a pesar del aspecto común mencionado anteriormente, que se manifiesta de manera específica en cada territorio. Es relevante indicar que los impactos en las regiones presentaron características de acuerdo a cada piso ecológico. Estudios anteriores han informado que las comunidades de Loreto están en riesgo de contraer enfermedades transmitidas por el agua debido a las inundaciones estacionales y la falta de sistemas de saneamiento adecuados (Faldetta et al. 2014). A través de los tres territorios, los y las participantes mostraron preocupación porque los cambios ambientales afectan la accesibilidad del agua para consumo humano. Por otro lado, existe una tensión constante en relación con el uso doméstico del agua para consumo humano, y para tareas productivas como la agricultura en las regiones de Piura y Cusco. Esta tensión se mostró más evidente en zonas con agricultura más intensiva como Piura (Mills-Novoa \& Hermoza 2017).

Las Naciones Unidas han desarrollado un plan de acción para el desarrollo sostenible que insta a los gobiernos a educar y empoderar a jóvenes, niños y niñas para que se comprometan con el cambio climático (Narksompong \& Limjirakan 2015). Bajo esta premisa, uno de los hallazgos más destacados de este trabajo fue el interés de las y los jóvenes y adolescentes de participar con su comunidad para prevenir los impactos actuales y potenciales del cambio climático. Es valioso contar con esta disposición, que debe traducirse no solo en reclutar los esfuerzos de estos grupos, sino, sobre todo, incorporar sus voces y necesidades en las actividades de adaptación y mitigación. Integrar su visión y fortalecer una mirada sistémica desde la base de su formación en la escuela es crucial para este objetivo (Schreiner et al. 2005). A solicitud de los propios jóvenes, estos conocimientos deben ser trasladados a aspectos diarios en los cuales puedan incidir y reforzar los conceptos aprendidos en las aulas, para traducirlo también en acciones de las cuales ellos quieren formar parte. Una revisión realizada anteriormente recogiendo la experiencia de los jóvenes, a nivel internacional, también había destacado el interés de estos por involucrarse en las soluciones orientadas a mitigar y adaptarse al cambio climático (Lee et al. 2020). 


\section{Conclusiones y recomendaciones}

Los participantes no identificaron el concepto de cambio climático, sin embargo, reconocieron cambios persistentes en el clima que afectan su vida diaria. Esta situación debe convertirse en una opción de acceso para incorporar los conceptos de cambio climático y las políticas públicas necesarias para la adaptación y mitigación. El interés y manifestación explícita de los jóvenes para su participación en estas actividades debe ser un activo para incorporar su visión y sus necesidades dentro de las políticas públicas necesarias para enfrentar la crisis climática.

Un punto transversal fue el impacto en el acceso y calidad del recurso del agua, reconociendo el impacto en su salud, sistemas de producción agrícola y fuentes de contaminación del recurso. Existe una inequidad manifiesta que se ha evidenciado más en los meses de pandemia (Bowleg 2020). Estas desigualdades sociales y económicas también se presentan y serán evidentes con la actual crisis climática y con el acceso inadecuado a un recurso básico como el agua (Singer 2019). Lo más importante es que la gente «sobre el terreno» percibe estas desigualdades.

A manera de resumen, un representante de una organización ambiental en Loreto afirmó que «En este lío nosotros no somos los provocadores; sin embargo, seremos las víctimas». Esta frase sintetiza su sentir al tratarse de una región que, hasta el momento, no había contribuido significativamente al cambio climático, pero sí empieza a sentir el impacto del mismo. Asimismo, recoge la urgencia con la cual se debe actuar, priorizando iniciativas y proyecciones futuras de acuerdo con distintos escenarios frente al cambio climático. Estas actividades inmediatas deben tomar en cuenta las voces de los niños, niñas y adolescentes para la incorporación de políticas públicas sostenibles a nivel país. 


\section{Referencias}

Andersen, L. E., Suxo, A. \& Verner, D. (2009). Social Impacts of Climate Change in Peru: A District Level Analysis of the Effects of Recent and Future Climate Change on Human Development and Inequality. World Bank Policy Research Working Paper nro. 5091.

Bowleg, L. (2020). We're Not All in This Together: On COVID-19, Intersectionality, and Structural Inequality. American Journal of Public Health, 110(7), 917-917. https://doi.org/10.2105/AJPH.2020 .305766

Cunsolo Willox, A., Harper, S. L., Ford, J. D., Landman, K., Houle, K., \& Edge, V. L. (2012). «From this place and of this place:» Climate change, sense of place, and health in Nunatsiavut, Canada. Social Science \& Medicine, 75(3), 538-547. https://doi.org/10.1016/j.socscimed.2012. 03.043

Faldetta, K. F., Reighard, D.A., Dickinson, K. L.,Wang, C. Q., George, D. R., Rodriguez Benavides, L., \& Strosnider, W. H. J. (2014). Assessing domestic water quality in Belén municipality, Iquitos, Peru. Journal of Water, Sanitation and Hygiene for Development, 4(3), 391-399. https://doi.org/10.2166/washdev.2014.051

Fiksel, J. (2006). Sustainability and resilience: Toward a systems approach. Sustainability: Science, Practice and Policy, 2(2), 14-21. https://doi.org/10.1080/15487733.2006.11907980

Funatsu, B. M., Dubreuil, V., Racapé, A., Debortoli, N. S., Nasuti, S., \& Le Tourneau, F.-M. (2019). Perceptions of climate and climate change by Amazonian communities. Global Environmental Change, 57, 101923. https://doi.org/10.1016/j.gloenvcha.2019.05.007

Gatti, L. V., Basso, L. S., Miller, J. B., Gloor, M., Gatti Domingues, L., Cassol, H. L. G., Tejada, G., Aragão, L. E. O. C., Nobre, C., Peters, W., Marani, L., Arai, E., Sanches, A. H., Corrêa, S. M., Anderson, L., Von Randow, C., Correia, C. S. C., Crispim, S. P., \& Neves, R. A. L. (2021). Amazonia as a carbon source linked to deforestation and climate change. Nature, 595(7867), 388-393. https://doi.org/10.1038/s41586-021-03629-6

Hardoy, J., \& Romero Lankao, P. (2011). Latin American cities and climate change: Challenges and options to mitigation and adaptation responses. Current Opinion in Environmental Sustainability, 3(3), 158-163. https://doi.org/10.1016/j.cosust.2011. 01.004

Hissa, K. (2016). Using Photovoice to Understand Climate Change Adaptation in Rural Ontario. Geography and Environmental Studies Major Research Papers. https://scholars.wlu.ca/ges_mrp/6 
Lee, K., Gjersoe, N., O'Neill, S., \& Barnett, J. (2020). Youth perceptions of climate change: A narrative synthesis. WIREs Climate Change, 11(3). https://doi.org/10.1002/wcc.641

Mills-Novoa, M., \& Hermoza, R. T. (2017). Coexistence and conflict: IWRM and large-scale water infrastructure development in Piura, Peru. Water Alternatives, 10(2), 370-394. MINAM (2010). Plan de acción de adaptación y mitigación frente al cambio climático. https://sinia.minam.gob.pe/documentos/planaccion-adaptacion-mitigacion-frente-cambio-climatico

MINAM (2015). Estrategia Nacional ante el Cambio Climático. http://sigrid.cenepred.gob.pe/sigridv3/documento/1140

MINAM (2016). Informe: Tercera Comunicación Nacional del Perú a la Convención Marco de las Naciones Unidas sobre el Cambio Climático. SINIA | Sistema Nacional de Información Ambiental. Recuperado 21 de octubre de 2021, de https://sinia. minam.gob.pe/documentos/tercera-comunicacion-nacional-peruconvencion-marco-las-naciones

MINAM (s.f). Estrategia Nacional ante el Cambio Climático al 2050. Recuperado: 21 de octubre de 2021. https://www.gob.pe /institucion/minam/campa\%C3\%B1as/3453-estrategia-nacionalante-el-cambio-climatico-al-2050

MINAM \& MIMP (s.f.). Plan de Acción en Género y Cambio Climático. https://www.gob.pe/institucion/minam/informes-publicaciones /306199-plan-de-accion-en-genero-y-cambio-climatico

Narksompong, J., \& Limjirakan, S. (2015). Youth Participation in Climate Change for Sustainable Engagement: Youth Participation in Climate Change for Sustainable Engagement. Review of European, Comparative \& International Environmental Law, 24(2), 171-181. https://doi. org/10.1111/reel.12121

Ostapchuk, J., Harper, S., Cunsolo Willox, A., Edge, V. L., \& Community Government, R. I. (2015). Exploring Elders' and Seniors' Perceptions of How Climate Change is Impacting Health and Well-being in Rigolet,

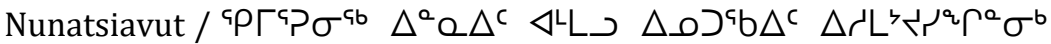

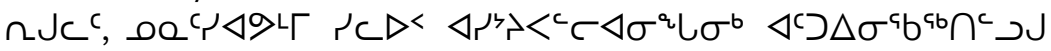

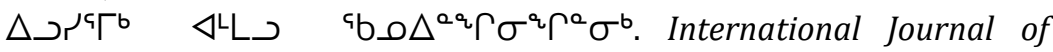
Indigenous Health, 9(2), 6. https://doi.org/10.18357/ijih92201214358 Sánchez, L. \& Reyes, O. (2015). Medidas de adaptación y mitigación frente al cambio climático en América Latina y el Caribe: Una revisión general. CEPAL. https://www.cepal.org/es/publicaciones/39781medidas-adaptacion-mitigacion-frente-al-cambio-climatico-americalatina-caribe 
Schreiner, C., Henriksen, E. K., \& Kirkeby Hansen, P.J. (2005). Climate Education: Empowering Today's Youth to Meet Tomorrow's Challenges. Studies in Science Education, 41(1), 3-49. https://doi.org/10.1080 $/ 03057260508560213$

Siclari, P. G. (2021). Amenazas de cambio climático, métricas de mitigación y adaptación en ciudades de América Latina y el Caribe. CEPAL. https:// www.cepal.org/es/publicaciones/46575-amenazas-cambio-climaticometricas-mitigacion-adaptacion-ciudades-america-latina

Singer, M. (2019). Climate change and social inequality: The health and social costs of global warming. Routledge/Taylor \& Francis Group.

Wang, C., \& Burris, M. A. (1997). Photovoice: Concept, Methodology, and Use for Participatory Needs Assessment. Health Education \& Behavior, 24(3),369-387.https://doi.org/10.1177/109019819702400 309

Watts, N., Amann, M., Arnell, N., Ayeb-Karlsson, S., Beagley, J., Belesova, K., Boykoff, M., Byass, P., Cai, W., Campbell-Lendrum, D., Capstick, S., Chambers,J.,Coleman,S.,Dalin,C., Daly,M.,Dasandi, N., Dasgupta,S., Davies, M., Di Napoli, C., ... Costello, A. (2021). The 2020 report of The Lancet Countdown on health and climate change: Responding to converging crises. The Lancet, 397(10269), 129-170. https://doi.org/10.1016/S0140-6736(20)32290-X 